Pacific Journal of Mathematics

LATTICE VERTEX POLYTOPES WITH INTERIOR LATTICE 


\title{
LATTICE VERTEX POLYTOPES WITH INTERIOR LATTICE POINTS
}

\author{
Douglas Hensley
}

\begin{abstract}
Consider a convex polytope with lattice vertices and at least one interior lattice point. We prove that the number of boundary lattice points is bounded above by a function of the dimension and the number of interior lattice points. This extends to arbitrary dimension a result of Scott for the two dimensional case.
\end{abstract}

Introduction. In real Euclidean space $\mathbf{R}^{D}$ of dimension $D$ there is the lattice $\mathbf{Z}^{D}$ of points with integer coordinates. Unless a different lattice is specified, a lattice point will mean a point of $\mathbf{Z}^{D}$, and a lattice simplex or lattice convex polytope will mean a simplex or convex polytope whose vertices are integer points, that is, elements of $\mathbf{Z}^{D}$. The interior in $\mathbf{R}^{D}$ of a set $S$ is denoted by $S^{\circ}$; if the affine span of $S$ has dimension less than $D$, we denote the relative interior of $S$ by $S^{\prime}$.

Consider a lattice convex polytope $P \subseteq \mathbf{R}^{D}$ with the number $K=$ $\#\left(P^{\circ} \cap \mathbf{Z}^{D}\right)$ of interior lattice points non-zero, and with a total of $J=\#\left(P \cap \mathbf{Z}^{D}\right)$ lattice points. Our principal result is that $J$ is bounded above by a function $B(K, D)$ of $K$ and $D$ alone.

For the case of zero symmetric convex polytopes $P$ there is no need to assume that the vertices are lattice points. By Van der Corput's generalization of Minkowski's theorem $\operatorname{vol}(P) \leq K \cdot 2^{D}[4]^{40}+\dagger$ By a theorem of Blichfeldt, if the lattice points of $P$ span $\mathbf{R}^{D}, J \leq D+D$ ! $\operatorname{vol}(P)[1]^{55}$. Otherwise we can consider a subspace of $\mathbf{R}^{D}$ and get the same inequality $J \leq D+D ! K \cdot 2^{D}$. On the other hand if $P$ need not be symmetric or have lattice point vertices then even for $D=2$ and $K=1, J$ can be arbitrarily large. For instance, $P$ might be the convex hull of $(-n, 0),\left(0,1+1 / n^{2}\right)$, $(n, 0)$. With the restriction to lattice point vertices and $D=2$ we have Scott's result that $J \leq 3 K+7(3 K+6$ for $K>1)$, and of course when $D=1$ we have trivially $J \leq K+2$. These three bounds are best possible. Our results are far from best possible, but in any case the largest possible $J$ grows rapidly with $D$, even for $K=1$. Zaks, Perles and Wills have given examples of lattice simplices in $\mathbf{R}^{D}$ for which $K=1$ and $J>2^{2^{D-1}}[\mathbf{1 1}]$. There are some grounds for the belief that these examples are best possible. (See §4.) The existence of $B(K, D)$ will follow from some facts about Diophantine approximation which we now establish.

$\dagger$ Here the number above the brackets gives the page number on which this result is found in Lekkerkerker [7]. 
2. Number theory. We start with a well-known approximation lemma.

LEMMA 2.1. Given a vector $\vec{v}=\left(v_{1}, v_{2} \cdots v_{D}\right) \in \mathbf{R}^{D}$ and an integer $T>0$ there exist integers $a_{1}, a_{2} \cdots a_{D}, b$ such that $1 \leq b \leq T^{D}$ and $\left|b v_{i}-a_{i}\right| \leq 1 / T$ for $1 \leq i \leq D$.

Proof. Consider the $T^{D}+1$ points $k \vec{v}, 0 \leq k \leq T^{D}$ reduced modulo 1 in each coordinate. Partitioning the unit cube $\left\{\vec{x}: 0 \leq x_{i} \leq 1\right.$ for $1 \leq i \leq$ $D$ ) into $T^{D}$ cubes of side $1 / T$, we conclude from the Dirichlet box principle that some two of them, say $k_{1}$ and $k_{2}$ with $k_{1}>k_{2}$, lie in the same small cube. Let $b=k_{1}-k_{2}$ and let $a_{i}$ be the integer nearest $b v_{i}$ for $1 \leq i \leq D$.

LEMMA 2.2. Let $\vec{w}=\left(w_{1}, w_{2} \cdots w_{D}\right)$ such that $\Sigma_{1}^{D} w_{l}=1$ and each $w_{i}>0$, and let $T>D$. Then there exist integers $P_{1}, P_{2} \cdots P_{D}, Q=\Sigma_{1}^{D} P_{l}$ such that $1 \leq Q \leq T^{D-1}, P_{l} \geq 0$ for $1 \leq i \leq D,\left|Q w_{1}-P_{1}\right| \leq D / T$ and $\left|Q w_{\imath}-P_{\imath}\right| \leq 1 / T$ for $2 \leq i \leq D$.

Proof. We write $\vec{w}=\vec{e}_{1}+\sum_{2}^{D} w_{i}\left(\vec{e}_{i}-\vec{e}_{1}\right)$. By Lemma 1 there exists $Q$, $1 \leq Q \leq T^{D}$, and $P_{2}, P_{3} \cdots P_{D}$ such that $\left|Q w_{i}-P_{i}\right| \leq 1 / T(2 \leq i \leq D)$. Since each $w_{l}>0, Q w_{i}>0$ so $P_{i} \geq 0$ for $i \geq 2$.

Let $P_{1}=Q-\Sigma_{2}^{D} P_{i}$. Then $\left|P_{1}-Q w_{1}\right|=\left|\Sigma_{2}^{D} P_{l}-Q \Sigma_{2}^{D} w_{i}\right|<D / T<$ 1 so that also $P_{1} \geq 0$.

LemMa 2.3. For each integer $D \geq 1$ there exists $\varepsilon(D)>0$ such that if $\vec{\alpha}=\left(\alpha_{1}, \alpha_{2} \cdots \alpha_{D}\right)$, each $\alpha_{i}>0$ and $1>\Sigma_{1}^{D} \alpha_{\imath}>1-\varepsilon(D)$ then there exist integers $Q \geq 1$ and $P_{1}, P_{2} \cdots P_{D} \geq 0$ such that $\Sigma_{1}^{D} P_{i}=Q$ and $(Q+1) \alpha_{i}>$ $P_{i}$ for each $i, 1 \leq i \leq D$.

Proof. For $D=1$ thus just says that there is an integer $Q$ such that $(Q+1) \alpha_{1}>Q$, so that we may take $\varepsilon(1)=1 / 2$. Now suppose $D>1$ and the lemma holds for $D-1$. Let $\vec{\alpha}=\left(\alpha_{1}, \ldots, \alpha_{D}\right)$ and without loss of generality assume $\alpha_{1} \geq \alpha_{2} \cdots \geq \alpha_{D}>0$. We want to choose $\varepsilon(D)$ in terms of $\varepsilon(D-1)$ so that if $1>\Sigma_{1}^{D} \alpha_{i}>1-\varepsilon(D)$ then the $P_{1}, \ldots, P_{D}$ and $Q$ of Lemma 2.3 exist. We choose it this way: Let

$$
T=\max \left\{1+\left[4\left(\varepsilon_{D-1}\right)^{-1}\right], 4 D^{2}+4 D+1\right\} .
$$

Let $\varepsilon(D)(>0)$ be $\min \left\{\frac{1}{2} \varepsilon(D-1),(D-1)^{-1}, \frac{1}{4} T^{1-D}\right\}$. Let $w_{i}=$ $\alpha_{i}(1-\varepsilon)^{-1}$ where $\varepsilon=1-\Sigma_{1}^{D} \alpha_{i}<\varepsilon(D)$.

By Lemma 2.2 there exist $P_{1}, P_{2}, \ldots, P_{D} \geq 0$ and $Q=\Sigma_{1}^{D} P_{i}$ such that $1 \leq Q \leq T^{D-1}$ and $\left|Q w_{1}-P_{1}\right| \leq D / T,\left|Q w_{l}-P_{i}\right| \leq 1 / T$ for $2 \leq i \leq D$. 
Now for $2 \leq i \leq D$,

$$
\begin{aligned}
(Q+1) \alpha_{i}- & P_{i}=\alpha_{i}+Q \alpha_{i}-P_{i}=\alpha_{i}+Q \alpha_{i}-Q w_{i}+Q w_{i}-P_{i} \\
& \geq \alpha_{i}-Q \alpha_{i}(1 /(1-\varepsilon)-1)-1 / T \\
& >\alpha_{i}-Q \alpha_{i}(1 /(1-\varepsilon(D))-1)-1 / T \\
& \geq \alpha_{i}(1-2 Q \varepsilon(D))-1 / T \geq \alpha_{i}\left(1-2 T^{D-1} \varepsilon(D)\right)-1 / T .
\end{aligned}
$$

If now $\alpha_{i} \geq \frac{1}{2} \varepsilon(D-1)$ this last is positive, from the definitions of $T$ and $\varepsilon(D)$. If $\alpha_{i}<\frac{1}{2} \varepsilon(D-1)$ then $\alpha_{D}<\frac{1}{2} \varepsilon(D-1)$ so that $\sum_{1}^{D-1} \alpha_{i}>1-\varepsilon(D)$ $-\frac{1}{2} \varepsilon(D-1) \geq 1-\varepsilon(D-1)$. In this case the $P_{1}, \ldots, P_{D-1}, Q$ guaranteed by Lemma 2.2 (assumed true for $D-1$ ) can be extended with $P_{D}=0$.

The case $i=1$ is a little different. Here we have $\alpha_{1} \geq 1 /(D+1)$ since $\varepsilon<\varepsilon(D) \leq 1 /(D+1)$, and we need $\frac{1}{2} \alpha_{1}\left(1-2 T^{D-1} \varepsilon(D)\right)>D / T$, which follows from $T>4 D(D+1)$.

We can determine the best constants $\varepsilon(D)$ in Lemma 2.3 for $D=1,2$ or 3. As noted, we can take $\varepsilon(1)=1 / 2$. No larger choice is possible because if $\alpha_{1}=1 / 2,(Q+1) \alpha_{1}>Q$ has no positive integer solution.

For $D=2$ and $\alpha_{1} \geq \alpha_{2}$ if $\alpha_{1}>1 / 2$ we take $Q=1, P_{1}=1$ and $P_{2}=0$, while if $\alpha_{2}>1 / 3, Q=2, P_{1}=P_{2}=1$. Thus we may take $\varepsilon(2)=$ $1-1 / 2-1 / 3=1 / 6$. For $D=3$ we can prove by such considerations that $\varepsilon(3)$ can be taken $=1 / 42$. For if $\alpha_{1}+\alpha_{2}+\alpha_{3}>41 / 42$ while $\alpha_{1} \leq$ $1 / 2$ and $\alpha_{2} \leq 1 / 3$ then $\alpha_{3}>1 / 7$. Now if $7\left(\alpha_{1}, \alpha_{2}, \alpha_{3}\right) \ngtr(3,2,1)$ (coordinatewise), then either $\alpha_{1} \leq 3 / 7$ or $\alpha_{2} \leq 2 / 7$. Either way, $\alpha_{3}>1 / 7+$ $1 / 21=4 / 21$. Eventually one arrives at $\alpha_{3}>1 / 4$, and then $4\left(\alpha_{1}, \alpha_{2}, \alpha_{3}\right)$ $>(1,1,1)$.

For $D=1,2$ or 3 these $\varepsilon(D)$ are best possible (consider $\alpha_{1}=1 / 2$, $\alpha_{2}=1 / 3$ and $\alpha_{3}=1 / 7$ ). For $D \geq 4$ this approach seems to break down.

In the next lemma we treat the case $K>1$.

Lemma 2.4. For integers $K \geq 2, D \geq 1$ there exists $\varepsilon(K, D)>0$ such that if $1>\Sigma_{1}^{D} \alpha_{i}>1-\varepsilon(K, D)$ and each $\alpha_{i}>0$ then there exist integers $P_{1}, P_{2} \cdots P_{D} \geq 0$ and $Q=\Sigma_{1}^{D} P_{i} \geq 1$ such that $(K Q+1) \alpha_{i}>K P_{i}$ for $1 \leq$ $i \leq D$.

Proof. For $D=1$ this says simply that if $\alpha<1$ is sufficiently large then there exists $Q \geq 1$ such that $(K Q+1) \alpha_{1}>K Q$, and we take $\varepsilon(K, 1)$ $=1 /(K+1)$. We now prove Lemma 2.4 for fixed $K$ by induction on $D$. Suppose it holds for $D-1$. Let $\vec{\alpha}=\left(\alpha_{1}, \alpha_{2} \cdots \alpha_{D}\right)$ with each $\alpha_{i}>0$ and $\Sigma_{1}^{D} \alpha_{i}=1-\varepsilon, \varepsilon>0$. If $\alpha_{D}<\varepsilon(K, D-1)-\varepsilon$ then $\sum_{1}^{D-1} \alpha_{\imath}>1-$ $\varepsilon(K, D-1)$ so we can use $P_{1}, P_{2} \cdots P_{D-1}, 0$ and $Q$ as in Lemma 2.3. 
Otherwise we use Lemma 2.2. Let

$$
T=\max \left\{1+\left[4 K(\varepsilon(K, D-1))^{-1}\right], 4 D^{2}+4 D+1\right\} .
$$

Let

$$
\varepsilon(K, D)=\min \left\{1 / 4 D^{2}, \frac{1}{4} \varepsilon(K, D-1), \varepsilon(1, D),(4 K)^{-1} T^{1-D}\right\} .
$$

For $2 \leq i \leq D$,

$$
(K Q+1) \alpha_{i}-K P_{i}=\alpha_{i}+K\left(Q \alpha_{i}-P_{i}\right) \geq \alpha_{i}(1-2 K Q \varepsilon)-K / T,
$$

with $Q \leq T^{D-1}$. This then is $>\frac{1}{2} \varepsilon(K, D-1)\left(1-2 K T^{D-1} \varepsilon(K, D)\right)$ $-K / T$. By the choice of $\varepsilon(K, D),\left(1-2 K T^{D-1} \varepsilon(K, D)\right)<1 / 2$, and by the choice of $T, \frac{1}{4} \varepsilon(K, D-1)>K / T$.

For $i=1$ we have $\alpha_{1} \geq(D+1)^{-1}$ so we need $\frac{1}{2}(D+1)^{-1}\left(\frac{1}{2}\right)>$ $K D / T$, which still follows from $T>4 D(D+1)$.

REMARK. The growth of $(\varepsilon(D))^{-1}$ is about like $2^{(D !)}$. The example of [11] has a simple variant with $\varepsilon$ like $2^{2^{D}}$. So bound and example have asymptotic $\log \log \log$ 's.

3. Geometry. Suppose now that $S$ is a simplex with vertices $0, X_{1}, X_{2} \cdots X_{D} \in \mathbf{Z}^{D}$ and an interior lattice point $Y=\Sigma_{1}^{D} \alpha_{l} X_{i}$.

Lemma 3.1. If $\Sigma_{1}^{D} \alpha_{i}>1-\varepsilon(K, D)$ then there are at least $K+1$ integer lattice points in $S^{\circ}$.

Proof. Apply Lemma 2.3 or 2.4. The points $Z_{k}=(k Q+1) Y-$ $k \sum_{l=1}^{D} P_{l} X_{i}$ are lattice points, distinct, and interior to $S$, for $0 \leq k \leq K$.

By translation we can make any vertex of a simplex be zero. This, with Lemma 3.1, gives

THEOREM 3.1. Suppose $S$ is simplex in $\mathbf{R}^{D}$ with integer lattice vertices $X_{0}, X_{1} \cdots X_{D}$ and exactly $K$ interior lattice points $Y_{j}, 1 \leq j \leq K, Y_{j}=$ $\sum_{i=0}^{D} \alpha_{\imath j} X_{\imath}$ with $\alpha_{i j}>0, \sum_{i=1}^{D} \alpha_{i j}=1$. Then for each $i$ and $j, \varepsilon(K, D) \leq$ $\alpha_{i j} \leq 1-D \varepsilon(K, D)$.

Corollary 3.2. Suppose $F$ is a lattice convex polytope in $\mathbf{R}^{D}$ of spanning dimension $D-1$, and lattice vertices $X_{1}, X_{2} \cdots X_{M}$. Let $X_{0}$ be a lattice point not in the span of $F$, and let $P$ be the conical polytope with $X_{0}$ the tip and $F$ the opposite face. If $\sharp\left(P^{\circ} \cap \mathbf{Z}^{D}\right)=K \geq 1$ then in any barycentric representation $Y=\sum_{0}^{M} \alpha_{i} X_{i}$ of an interior point of $P$ we have $\alpha_{0} \geq \varepsilon(K, D)$. 
Proof. By Caratheodory's theorem [3] there are $E \leq D$ vertices of $F$, say $V_{1}, V_{2} \cdots V_{E}$ such that $Y$ is in the relative interior of the simplex $S$ with vertices $X_{0}, V_{1} \cdots V_{E}$. Every lattice point in $S^{\prime}$ is also in $P^{\circ}$ (proof follows), so there are no more than $K$ in $S^{\prime}$. By Theorem 1, if $Y=\beta_{0} X_{0}+$ $\Sigma_{1}^{E} \beta_{i} V_{i}$ then $\beta_{0} \geq \varepsilon(K, D)$. But $\beta_{0}=\alpha_{0}$, since it is the ratio of the length of $\overline{Y Z}$ to $\overline{X_{0} Z}$, where $Z$ is the intersection of the line through $X_{0}$ and $Y$ with $F$.

We now prove that $S^{\prime} \subseteq P^{\circ}$.

Lemma 3.3. If $C$ is a convex set in $\mathbf{R}^{D}, Y \in C^{\circ}$ and $W_{0} \cdots W_{E}$ form the vertices of a simplex $W$ in $C$, with $E \leq D$ and $Y \in W$, then $W^{\prime} \subseteq C^{\circ}$.

Proof. Since $Y \in C^{\circ}$ there exists $\varepsilon>0$ such that if $\|\vec{U}\| \leq 1$ and $|\theta| \leq \varepsilon$ then $Y+\theta U \in C$. Write $Y$ as $\Sigma_{0}^{E} \alpha_{i} W_{i}, \alpha_{i}>0, \Sigma_{0}^{E} \alpha_{i}=1$. If $Z \in W^{\prime}=\Sigma_{0}^{E} \beta_{i} W_{i}$ with $\beta_{i}>0$ and $\Sigma_{0}^{E} \beta_{i}=1$ then there exists $\delta>0$ such that $\beta_{i}>\delta \alpha_{i}$ for $0 \leq i \leq E$. Now $Z+\theta \delta U=\Sigma_{0}^{E}\left(\beta_{i}-\delta \alpha_{i}\right) W_{i}+$ $\delta(Y+\theta U)$ is a convex positive combination of elements of $C$, so it is in C.

Until now it has been convenient to have the fixed lattice $\mathbf{Z}^{D}$ in mind, but all the results are equally true for any full lattice $L$ in $\mathbf{R}^{D}$, as there is a nonsingular linear transformation $\Phi: \mathbf{R}^{D} \rightarrow \mathbf{R}^{D}$ which maps $\mathbf{Z}^{D}$ onto $L$ while preserving barycentric coordinates, interiors and relative interiors, etc. We use this device to give an upper bound for the volume of an integer lattice simplex $S$ with $\sharp\left(Z^{D} \cap S^{\circ}\right)=K \geq 1$. Without loss of generality take 0 as one vertex of $S$, and let $\Phi$ be a linear transformation which takes $S$ onto the "standard simplex" $H$ with vertices $0, \vec{e}_{1}, \ldots, \vec{e}_{D}$, where $\vec{e}_{i}$ is the $i$ th unit coordinate vector in $\mathbf{R}^{D}$. Then $\Phi$ takes the lattice $\mathbf{Z}^{D}$ to a new lattice $L$, and the norm of $L,|L|$ is $|\operatorname{det} \Phi|$, and $\operatorname{vol}(S)=1 / D !\left|\operatorname{det} \Phi^{-1}\right|$. Thus any lower bound for $|L|$ gives an upper bound for $\operatorname{vol}(S)$. Suppose $Y_{1} \in S^{\circ} \cap \mathbf{Z}^{D}, Y_{1}=\Sigma_{1}^{D} \alpha_{i} X_{i}$. Let $V_{1}=\Phi Y_{1}$ $=\sum_{1}^{D} \alpha_{i} \vec{e}_{i}$. Given $U=\sum_{1}^{D} u_{i} \vec{e}_{i}$ with $\left|u_{i}\right|<\alpha_{i}$, either $V_{1}+U \in H^{\circ}$ or $V_{1}-U \in H^{\circ}$, since $\alpha_{i} \pm u_{i}>0$ and one of $\Sigma_{1}^{D}\left(\alpha_{i}+u_{i}\right), \Sigma_{1}^{D}\left(\alpha_{i}-u_{i}\right)$ is less than 1.

By Van der Corput's theorem the region $\left\{V_{1}+U:\left|u_{i}\right|<\alpha_{i}, 1 \leq i \leq\right.$ $D\}$ contains at least $\left(\Pi_{1}^{D} \alpha_{\imath}\right)\left|\operatorname{det} \Phi^{-1}\right|$ pairs of points $V_{1} \pm U \in L$. Of each pair at least one is in $H^{\circ}$. Thus $K=\sharp\left(S^{\circ} \cap \mathbf{Z}^{D}\right)=\sharp\left(H^{\circ} \cap L\right) \geq$ $\left(\Pi_{1}^{D} \alpha_{\imath}\right)\left|\operatorname{det} \Phi^{-1}\right|, \geq(\varepsilon(K, D))^{D}\left|\operatorname{det} \Phi^{-1}\right|$ by Theorem 3.1. So $|\operatorname{det} \Phi|$ $\geq(\varepsilon(K, D))^{D} K^{-1}$. Since $|\operatorname{det} \Phi|=\operatorname{vol} H /$ vol $S$, we have vol $S \leq$ $(D !)^{-1} K(\varepsilon(K, D))^{-D}$. We summarize this in

THEOREM 3.4. Suppose $S$ is a simplex in $\mathbf{R}^{D}$ with vertices in $\mathbf{Z}^{D}$, and let $K=\sharp\left(S^{\circ} \cap \mathbf{Z}^{D}\right)$. If $K \geq 1$ then vol $S \leq(D !)^{-1} K(\varepsilon(K, D))^{-D}$. 
REMARK. We could get a better lower bound for $\Pi_{1}^{D} \alpha_{i}$ by using the fact that not only is each $\alpha_{i} \geq \varepsilon(K, D)$, but (perhaps renaming some vertices) $\Sigma_{1}^{D} \alpha_{i} \approx 1$ yet $\Sigma_{1}^{E} \alpha_{i} \leq 1-\varepsilon(K, E)$ for $E<D$. With such a weak bound for $\varepsilon(K, D)$, though, this seems pointless.

A theorem of Blichfeldt says that if a convex body $P$ in $\mathbf{R}^{D}$ has $J=\#\left(\mathbf{Z}^{D} \cap P\right)>D$ lattice points, spanning $\mathbf{R}^{D}$, then $\operatorname{vol}(P) \geq$ $(J-D) / D$ ! [1], or equivalently $J \leq D+D$ ! $\operatorname{vol}(P)$. Thus we get the

COROLlaRY 3.5. Under the hypotheses of Theorem 3.4, $\sharp\left(S \cap \mathbf{Z}^{D}\right) \leq$ $D+K(\varepsilon(K, D))^{-D}$.

For a general convex polytope $P$ with vertices in $\mathbf{Z}^{D}$ and $K \geq 1$ lattice points in $P^{\circ}$, from Corollary 3.2 we have that the coefficient $\sigma$ of asymmetry about any of the interior lattice points is $\leq(1-\varepsilon(K, D)) / \varepsilon(K, D)$. When $K=1$ we have by a theorem of Mahler (Sawyer gives a little sharper version) $[8,9]^{45}$ that $V(P) \leq(\varepsilon(D))^{-D}$. The proof of Mahler's theorem given in [7] ${ }^{45}$ uses Blichfeldt's theorem [2] ${ }^{35}$ that a region of volume $>1$ contains two points $x, y$ congruent modulo $\mathbf{Z}^{D}$. Van der Corput [4] ${ }^{40}$ generalized this to say that a region of volume $>K$ contains $K+1$ points congruent modulo $\mathbf{Z}^{D}$. If we use this in place of Blichfeldt's result we get an analogous generalization of Mahler's theorem. From it we conclude that for arbitrary $K \geq 1$,

$$
\operatorname{vol}(P) \leq K(\varepsilon(K, D))^{-D} .
$$

This and a corollary complete the story.

THEOREM 3.6. Let $P$ be a convex polytope in $\mathbf{R}^{D}$ with vertices in $\mathbf{Z}^{D}$ and with $K=\#\left(P^{\circ} \cap \mathbf{Z}^{D}\right) \geq 1$. Then $\operatorname{vol}(P) \leq K(\varepsilon(K, D))^{-D}$.

Corollary 3.7. If $J=\sharp\left(P \cap \mathbf{Z}^{D}\right)$ then $J \leq D+K(D !)(\varepsilon(K, D))^{-D}$.

4. Toward best possible results. Here we indicate some reasons for our belief that the examples of [11] with $K=1$ and $D \geq 3$ are best possible. Suppose $S$ is a lattice simplex with lone interior point $Y=$ $\Sigma_{0}^{D} \alpha_{\imath} X_{i}$, where $X_{0}, \ldots, X_{D}$ are the vertices of $S$ and $\alpha_{1} \geq \cdots \geq \alpha_{D} \geq \alpha_{0}$. We proved in $\$ 2$ that for arbitrary $D, \alpha_{1}+\alpha_{2} \leq 5 / 6$, and $\alpha_{1}+\alpha_{2}+\alpha_{3} \leq$ $41 / 42$. For $D=4$, if $\Sigma_{1}^{4} \alpha_{i}>1805 / 1806$ then $\alpha_{4}>1 / 43$. The minimum of $\alpha_{1} \alpha_{2} \alpha_{3} \alpha_{4}$ subject to $\Sigma_{1}^{4} \alpha_{i} \geq 1805 / 1806, \Sigma_{1}^{3} \alpha_{i} \leq 41 / 42, \Sigma_{1}^{2} \alpha_{i} \leq 5 / 6$ and $\alpha_{1} \leq 1 / 2,0<\alpha_{4} \leq \alpha_{3} \leq \alpha_{2} \leq \alpha_{1}$ is $1 / 1806$, by elementary calculus. Since $\operatorname{Norm}(L) \geq 1 / 1806$ and $\operatorname{vol}\left(\vec{e}_{1}, \vec{e}_{2}, \vec{e}_{3}, \vec{e}_{4}, \Phi \vec{Y}\right)\{$ simplex $\}$ is $\frac{1}{4 !}\left(1-\Sigma_{1}^{4} \alpha_{i}\right)$ $\geq \frac{1}{4 !} \operatorname{Norm}(L), \Sigma_{1}^{4} \alpha_{i} \leq 1805 / 1806$. This proves that for $D=3$, (4) the 
simplex with vertices $0,2 \vec{e}_{1}, 3 \vec{e}_{2}, 7 \vec{e}_{3},\left(43 \vec{e}_{4}\right)$ has maximal coefficient $\sigma$ of asymmetry about $Y$. Unfortunately it does not show that for arbitrary $D$, $\Sigma_{1}^{4} \alpha_{i} \leq 1805 / 1806$.

For any $D$, the $\alpha_{i}$ must be rational. For let $\Lambda^{\prime}$ be the lattice generated by $\left\{X_{i}-X_{0}, 1 \leq i \leq D\right\}$. If some $\alpha_{i}$ were irrational there would be infinitely many points of $\Lambda$ in a fundamental cell of $\Lambda^{\prime}$ since no two $n\left(Y-X_{0}\right), n \geq 1$, would be congruent $\bmod \Lambda^{\prime}$. But $\Lambda$ is discrete so this is impossible. So let $\alpha_{i}=v_{l} / x_{l}, 0 \leq i \leq D$, with $v_{i}, x_{i}>0$ and $\operatorname{gcd}\left(v_{i}, x_{i}\right)=$ 1 for $0 \leq i \leq D$ ).

The numbers $2,3,7,43$ in the simplex examples for $D=3$ or 4 are the start of a well-known sequence given recursively by $y_{1}=2, y_{n+1}=$ $y_{n}^{2}-y_{n}+1$ for $n \geq 1$. The $y_{i}$ 's are pairwise relatively prime, and $\Sigma_{1}^{D} y_{l}^{-1}$ $=1-\left(y_{D+1}-1\right)^{-1}<1$. Thus the lattice simplex $S_{D}$ with vertices 0 and $y_{l} \vec{e}_{i}, \quad 1 \leq i \leq D$ has the single interior lattice point $Y_{D}=\Sigma_{1}^{D} \vec{e}_{i}$. This example (here slightly modified) is first given in [11] and has at least $2^{2^{D-1}}$ boundary lattice points. We believe it to be best possible in the sense that the coefficient $\sigma_{D}$ of asymmetry for $S_{D}$ about $Y_{D} \geq \sigma$ for any other lattice simplex $S$ with lone interior lattice $Y$, about $Y$.

Let $S$ be such a simplex, and $Y=\Sigma_{0}^{D} \alpha_{i} X_{i}=\Sigma_{0}^{D}\left(v_{l} / x_{l}\right) X_{i}$ as before, with $\alpha_{1} \geq \alpha_{2} \geq \cdots \geq \alpha_{D} \geq \alpha_{0}>0$. With the additional assumption that $\left(x_{1}, x_{2}, \ldots, x_{D}\right)$ are pairwise relatively prime we can prove this conjecture, or what is the same, the following theorem.

THEOREM 4.1. Suppose $\left(x_{1}, x_{2}, \ldots, x_{D}\right)$ are pairwise relatively prime. Then $\Sigma_{1}^{D} v_{i} / x_{\imath} \leq \Sigma_{1}^{D} 1 / y_{i}$.

Conjecture. This holds whether or not the $x_{i}$ 's are pairwise relatively prime. (We have seen so for $1 \leq D \leq 4$.)

We begin the proof of Theorem 4.1 with an old Egyptian fractions result.

LEMMa 4.1. (Curtis [5], Erdös [6].) Let $x_{1}, x_{2} \cdots x_{D}$ be positive integers. If $\Sigma_{1}^{D}\left(1 / x_{i}\right)<1$ then $\Sigma_{1}^{D}\left(1 / x_{i}\right) \leq \Sigma_{1}^{D}\left(1 / y_{i}\right)=1-\prod_{1}^{D} y_{i}^{-1}=1-$ $\left(y_{D+1}-1\right)^{-1}$.

Let $\varepsilon_{k}=\left(y_{k+1}-1\right)^{-1}$.

Lemma 4.2. For every $K, D \geq 1$ if $\left(v_{l}, x_{l}\right), 1 \leq i \leq D$ are $D$ pairs of relatively prime positive integers, and if $1-\varepsilon_{D+K-1}<\Sigma_{1}^{D}\left(v_{i} / x_{i}\right)<1$ then $\Sigma_{1}^{D} v_{i} \geq D+K$. 
Proof. (I. Borosh, private communication.) If each $v_{i} / x_{t}$ is replaced with $v_{i}$ copies of $1 / x_{i}$ there are then at least $D+K$ Egyptian fractions in the sum, by Lemma 4.1.

LeMma 4.3. Let $D \geq 2, K, v_{1} \cdots v_{D}, x_{1} \cdots x_{D}$ be positive integers such that $\operatorname{gcd}\left(v_{l}, x_{i}\right)=1$ for $1 \leq i \leq D$ and $\operatorname{gcd}\left(x_{l}, x_{j}\right)=1$ for $1 \leq i<j \leq D$. Let $M=\Pi_{1}^{D} x_{i}$ and $A_{t}=M v_{i} / x_{i}, 1 \leq i \leq D$. Let $\alpha_{i}=v_{i} / x_{i}=A_{i} / M$ and suppose $\operatorname{gcd}\left(A_{D}, M\right) \leq \operatorname{gcd}\left(A_{i}, M\right), 1 \leq i<D$, or equivalently $x_{D} \geq x_{i}$. Let $\theta_{2}, \theta_{3} \cdots \theta_{K}$ be any $K-1$ rational numbers $0<\theta_{\imath}<1$. If

$$
1-\varepsilon_{D+K-1}<\sum_{1}^{D} \alpha_{i}<1
$$

then there exist positive integers $a_{1}, a_{2} \cdots a_{D}$, $m$ such that

(i) $a_{i} / m<\alpha_{i}$ for $1 \leq i \leq D$

(ii) $m \alpha_{D}-a_{D} \neq \theta_{j}$ for $2 \leq j \leq K$, and $m \alpha_{D}-a_{D} \neq \alpha_{D}$, and

(iii) $\Sigma_{1}^{D}\left(m A_{i}-M a_{i}\right)<M$.

Remark. For Theorem 4.1 we only need the case $K=1$.

Proof. By Lemma 4.2, $\Sigma_{1}^{D}\left(v_{i}-1\right) \geq K$. Since $\operatorname{gcd}\left(A_{D}, M\right) \leq$ $\operatorname{gcd}\left(A_{i}, M\right)$ for $i \neq D, x_{D} \geq x_{i}$ for $i \neq D$. Since $\Pi_{1}^{D}\left(1 / x_{\imath}\right) \leq 1-\Sigma_{1}^{D} v_{l} / x_{t}$ $<\varepsilon_{D+K-1}, x_{D}^{D} \geq\left(\varepsilon_{D+K-1}\right)^{-1}$ and $x_{D}>K+1$. For it is readily seen that $\varepsilon_{l}^{-1} \geq 2^{2^{i-1}}$ for $i \geq 1$, and $D-\log _{2} D \geq 1, K-(\log \log )_{2} K \geq 2$ so that $D+K-2 \geq 1+\log _{2} D+(\log \log )_{2} K$ and $2^{2^{D+K-2}} \geq K^{2 D}>K+1$ for $K>1$, while for $K=1$, we have directly $\varepsilon_{D}^{-1}>2$ since already $\varepsilon_{2}^{-1}=6$. Now by the Chinese remainder theorem, for each integer $r, 1 \leq r \leq K+1$ there exists an $m>1$ such that $m v_{\imath} \equiv 1 \bmod x_{\imath}$ for $1 \leq i<D$ and $m v_{D} \equiv$ $r \bmod x_{D}$. (This is why we had to assume the $x_{i}$ relatively prime). Since $x_{D}>K+1$ these $K+1$ possibilities are distinct. Choose $r$ so that $r / x_{D}$ $\neq \alpha_{D}, \theta_{2}, \theta_{3} \cdots \theta_{K}$. Let $a_{i}=\left(m v_{i}-1\right) / x_{\imath}$ for $1 \leq i<D$, and $a_{D}=$ $\left(m v_{D}-r\right) / x_{D}$. These are integers because of the congruence conditions, and clearly (i) and (ii) are satisfied. Now since $x_{D} \geq x_{i}$ for $1 \leq i<D$, and since $\sum_{1}^{D} v_{i} \geq D+K$,

$$
(K+1) / x_{D}+\sum_{i=1}^{D-1}\left(1 / x_{\imath}\right) \leq \sum_{1}^{D}\left(v_{i} / x_{i}\right)<1
$$

implies that

$$
\sum_{1}^{D}\left(m v_{l} x_{l}^{-1}-a_{i}\right)=\left\{\sum_{1}^{D-1} 1 / x_{i}\right\}+r / x_{D}<1,
$$

which is equivalent to (iii). 
Suppose $0, X_{1} \cdots X_{D}$ are the vertices of $S$, and are in $\mathbf{Z}^{D}$. If $Y_{1}, Y_{2} \cdots Y_{K}$ are lattice points of $S^{\circ}$ and $Y_{1}=\Sigma_{1}^{D} \alpha_{i} X_{i}$ with relatively prime $x_{i}$, and if $\Sigma_{1}^{D} \alpha_{i}>1-\varepsilon_{D+K-1}$ then let $\theta_{j}, 2 \leq j \leq K$ be the $X_{D}$ coefficient of $Y_{j}$. Apply Lemma 4.3 and let $Y_{K+1}=m Y_{1}-\Sigma_{1}^{D} a_{i} X_{i}$. Then $Y_{K+1} \in S^{\circ}$ and different from $Y_{1} \cdots Y_{K}$ by Lemma 4.3. The case $K=1$ of these conclusions is Theorem 4.1.

REMARK. The estimate due to Borosh is not best possible. It would be interesting to know the maximum value of $\Sigma_{1}^{D} v_{i} / x_{i}$ subject to $0<v_{i} / x_{i}$, $\Sigma_{1}^{D} v_{i} / x_{i}<1$ and $\Sigma_{1}^{D} v_{i}=D+K-1$.

\section{REFERENCES}

1. H. F. Blichfeldt, A new principle in the geometry of numbers, with some applications, Trans. Amer. Math. Soc., 15 (1914), 227-235.

2. N Notes on geometry of numbers, Bull. Amer. Math. Soc., 27 (1921), 150-153.

3. C. Caratheodory. (See H. G. Eggleston, Convexity, Cambridge University Press (1958), p. 35.)

4. J. G. van der Corput, Verallgemeinerung einer Mordellschen Beweismethode in der Geometrie der Zahlen II, Acta Arith., 2 (1936), 145-146.

5. Curtis, On Kellogg's Diophantine Problem, Amer. Math. Monthly, 29 (1922), 380-387.

6. P. Erdös, $A z \frac{1}{x_{1}}+\frac{1}{x_{2}}+\cdots+\frac{1}{x_{n}}=\frac{a}{b}$ egyenletegész számú megoldásairól, Math. Lapok, (1950), 192-210.

7. C. G. Lekkerkerker, Geometry of Numbers, p. 35, 40, 45, and 55, Wolters-Noordhoff, Groningen, 1969.

8. K. Mahler, Ein Übertragungsprinzip für konvexe Körper, Časopis Pěst. Mat. Fyz., 68 (1939), 93-102.

9. D. B. Sawyer, The lattice determinants of asymmetric convex regions, J. London Math. Soc., 29 (1954), 251-254.

10. P. R. Scott, On convex lattice polygons, Bull. Austral. Math. Soc., 15 (1976), 395-399.

11. J. Zaks, M. A. Perles and J. M. Wills, On the lattice polytopes having interior lattice points, Elemente der Math., 37, No. 2 (1982), 44-46.

Received May 28, 1981 and in revised form November 2, 1981.

TEXas A \& M University

College Station, TX 77843 



\section{PACIFIC JOURNAL OF MATHEMATICS}

EDITORS

Donald BABBITT (Managing Editor)

University of California

Los Angeles, CA 90024

Hugo Rossi

University of Utah

Salt Lake City, UT 84112

C. C. Moore and Arthur Ogus

University of California

Berkeley, CA 94720
J. DugunduI

Department of Mathematics

University of Southern California

Los Angeles, CA 90089-1113

R. FinN and H. SAmelson

Stanford University

Stanford, CA 94305

\section{ASSOCIATE EDITORS}
R. ARENS
E. F. BECKENBACH
B. H. NEUMANN
F. WolF
K. YoshidA (1906-1982)

\section{SUPPORTING INSTITUTIONS}

UNIVERSITY OF ARIZONA

UNIVERSITY OF BRITISH COLUMBIA

CALIFORNIA INSTITUTE OF TECHNOLOGY

UNIVERSITY OF CALIFORNIA

MONTANA STATE UNIVERSITY

UNIVERSITY OF NEVADA, RENO

NEW MEXICO STATE UNIVERSITY

OREGON STATE UNIVERSITY
UNIVERSITY OF OREGON

UNIVERSITY OF SOUTHERN CALIFORNIA

STANFORD UNIVERSITY

UNIVERSITY OF HAWAII

UNIVERSITY OF TOKYO

UNIVERSITY OF UTAH

WASHINGTON STATE UNIVERSITY

UNIVERSITY OF WASHINGTON 


\section{Pacific Journal of Mathematics}

Vol. 105, No. 1 September, 1983

Kenneth F. Andersen, On the transformation of Fourier coefficients of

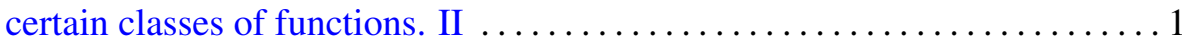

Gavin Brown, Irving Leonard Glicksberg and Edwin Hewitt, Indicator functions with large Fourier transforms $\ldots \ldots \ldots \ldots \ldots \ldots \ldots \ldots \ldots \ldots$

Shih-Sen Chang, Some random fixed point theorems for continuous random

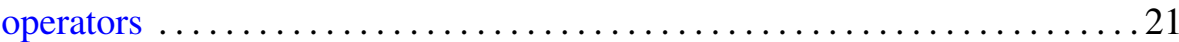

K. C. Chattopadhyay and Olav Njstad, Quasiregular nearness spaces and

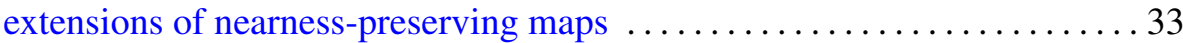

Thomas W. Cusick, The two-dimensional Diophantine approximation

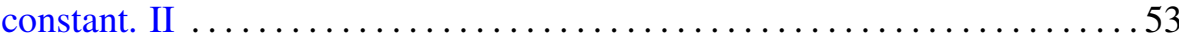

Eric Karel van Douwen and Jan van Mill, Spaces without remote points . . .669 Hector O. Fattorini, Convergence and approximation theorems for

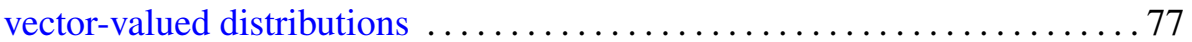

John J. F. Fournier and Louis Pigno, Analytic and arithmetic properties of

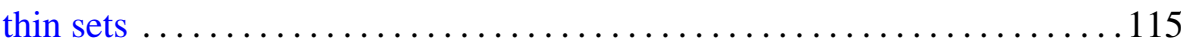

David Goss, On a new type of $L$-function for algebraic curves over finite

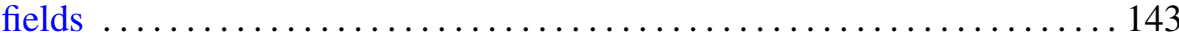

Douglas Austin Hensley, Lattice vertex polytopes with interior lattice

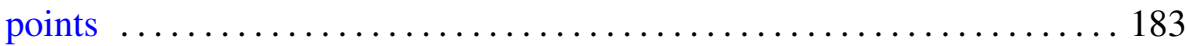

J. K. Kohli, Monotone extensions of mappings and their applications ...... 193

John C. Morgan, II, On equivalent category bases . . . . . . . . . . . . 207

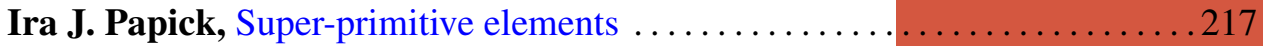

José Luis Rubio de Francia and José Luis Torrea, Vector extensions of

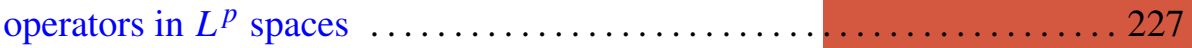

Mark Phillip Thomas, Closed ideals of $l^{1}\left(\omega_{n}\right)$ when $\left\{\omega_{n}\right\}$ is star-shaped $\ldots 237$ 